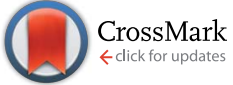

Cite this: Nanoscale, 2014, 6, 11387

Received 20th June 2014

Accepted 22nd July 2014

DOI: $10.1039 / c 4 n r 03456 b$

www.rsc.org/nanoscale

\title{
Study of exciton transfer in dense quantum dot nanocomposites $\uparrow$
}

\author{
Burak Guzelturk, ${ }^{\text {ab }}$ Pedro Ludwig Hernandez-Martinez, ${ }^{a b}$ Vijay Kumar Sharma, ${ }^{a}$ \\ Yasemin Coskun, ${ }^{a}$ Vusala Ibrahimova, ${ }^{a}$ Donus Tuncel, ${ }^{a}$ Alexander O. Govorov, ${ }^{c}$ \\ Xiao Wei Sun, ${ }^{\mathrm{b}}$ Qihua Xiong ${ }^{\mathrm{b}}$ and Hilmi Volkan Demir ${ }^{\star a b}$
}

\begin{abstract}
Nanocomposites of colloidal quantum dots (QDs) integrated into conjugated polymers (CPs) are key to hybrid optoelectronics, where engineering the excitonic interactions at the nanoscale is crucial. For such excitonic operation, it was believed that exciton diffusion is essential to realize nonradiative energy transfer from CPs to QDs. In this study, contrary to the previous literature, efficient exciton transfer is demonstrated in the nanocomposites of dense QDs, where exciton transfer can be as efficient as $80 \%$ without requiring the assistance of exciton diffusion. This is enabled by uniform dispersion of QDs at high density (up to $\sim 70 \mathrm{wt} \%$ ) in the nanocomposite while avoiding phase segregation. Theoretical modeling supports the experimental observation of weakly temperature dependent nonradiative energy transfer dynamics. This new finding provides the ability to design hybrid light-emitting diodes that show an order of magnitude enhanced external quantum efficiencies.
\end{abstract}

\section{Introduction}

Colloidal quantum dots (QDs) are prominent materials for optoelectronics with excellent prospects for light generation, ${ }^{\mathbf{1}}$ light harvesting, ${ }^{2}$ light detection, ${ }^{3}$ and lasing. ${ }^{4}$ Alternatively, conjugated polymers (CPs) are an important class of organic semiconductors that exhibit complementary properties..$^{5-7}$ Nanocomposites integrating both CPs and QDs potentially offer a mutual synergy. ${ }^{8-11}$ To this end, engineering excitonic interactions, which crucially depends on the interactions at the nanoscale, is vital for developing hybrid materials for high performance hybrid optoelectronic devices. ${ }^{11,12}$ Fluorescence resonance energy transfer (FRET) from CPs to QDs was shown to be possible in CP-QD composite systems, which were either mechanically blended or electrostatically integrated. ${ }^{13-19}$ For such energy transfer to occur, exciton diffusion in CPs was shown to be essentially required. ${ }^{20}$ However, exciton diffusion assisted FRET was demonstrated with only low density QD (3 wt\%) incorporated CP-QD blends in order to prevent phase

\footnotetext{
${ }^{a}$ Department of Electrical and Electronics Engineering, Department of Physics, Department of Chemistry, and UNAM-National Nanotechnology Research Center, Institute of Materials Science and Nanotechnology, Bilkent University, Ankara, TR-06800,Turkey.E-mail:volkan@bilkent.edu.tr; hvdemir@ntu.edu.sg

${ }^{b}$ Luminous! Center of Excellence for Semiconductor Lighting and Displays, School of Electrical and Electronic Engineering, School of Physical and Mathematical Sciences, Nanyang Technological University, Nanyang Avenue, Singapore 639798, Singapore ${ }^{c}$ Department of Physics and Astronomy, Ohio University, Athens, Ohio, 45701, USA $\dagger$ Electronic supplementary information (ESI) available: Information about the functionalized polymer structure, TEM of the QDs, XPS analysis of the nanocomposites, derivation of the energy transfer models and temperature dependent steady state PLs. See DOI: 10.1039/c4nr03456b
}

segregation, which occurs at increased densities of QDs in the CP-QD blends. ${ }^{\mathbf{2 0 2 1}}$ To date, interplay between exciton transfer and diffusion has not been understood, nor studied for high density QD loaded nanocomposites, which would be inevitable from the technological point of view for the realization of hybrid organic-inorganic optoelectronic devices.

In this work, we systematically study FRET in low and high density QD incorporated nanocomposites and blends achieved via utilization of two different CPs, i.e., functionalized and nonfunctionalized polyfluorene, respectively. These CPs have similar conjugated backbones but have different side chain groups, which possess different compatibility with QDs. The functionalized CP significantly suppresses phase separation in nanocomposite films when incorporated with high density QDs (up to $70 \mathrm{wt} \%$ ). On the other hand, nonfunctionalized CP exhibits severe phase segregation in blended films incorporating high density QDs. In these nanocomposites and blends, we investigate FRET at low and high QD density loadings as a function of temperature. In the case of low density QD incorporation, exciton diffusion assisted FRET is the dominant exciton transfer mechanism for both CPs. In the case of high density QD loadings; temperature dependent FRET kinetics are observed to substantially differ between the nanocomposite (functionalized CP-QDs) and the blend (nonfunctionalized CPQDs). The nanocomposite with high density QDs dominantly exhibits direct exciton transfer, which does not require exciton diffusion assistance. Whereas, the blend with high density QDs exhibits high temperature sensitivity for the FRET kinetics since exciton diffusion assistance is still required due to the aggregate formation of the QDs in blended films. These exciton diffusion 
assisted exciton transfer and direct exciton transfer channels are schematically summarized in Fig. 1a for the nanocomposite and blend cases at low and high density QDs. Through theoretical energy transfer models, we infer the cooperative and competitive nature between the exciton transfer and the exciton diffusion. In high density QD incorporated nanocomposites exciton diffusion is suppressed by 5 -fold as compared to the low density QDs. Finally, we demonstrate hybrid light-emitting diodes that employ the nanocomposites and the blends as the light-emitting layer of the devices. Nanocomposite-LEDs outperform other devices with a dominant QD emission in their electroluminescence spectra thanks to the efficient energy transfer pumping, whereas blend-LEDs always exhibit emission from the nonfunctionalized CP due to incomplete energy transfer to QDs.

\section{Experimental section}

\section{Synthesis of the functionalized polymer}

Poly[(9,9-bis\{propenyl\}fluorenyl-2,7-diyl)-co-(9,9-dihexylfluorenyl2,7-diyl)] (P1) was synthesized by the Suzuki coupling of 9,9dihexylfluorene-2,7-bis(trimethyleneborate) and 9,9-bis(propenyl)-2,7-dibromo-9H-fluorene and through the thiol-ene reaction allyl groups reacted with mercaptoacetic acid to afford poly[(9,9-bis $\{$ carboxymethylsulfonyl-propyl\}fluorenyl-2,7-diyl)-co-(9,9dihexylfluorenyl-2,7-diyl)] (functionalized CP). The conversion of allyl to a carboxymethylsulfonyl group was confirmed by the presence of characteristic carbonyl bond stretching at $1650 \mathrm{~cm}^{-1}$ in the IR spectrum of the functionalized CP. The weight average molecular weights $\left(M_{\mathrm{w}}\right)$ were determined to be $9.02 \times 10^{3} \mathrm{~g}$ $\mathrm{mol}^{-1}$ for P1 by gel permeation chromatography (GPC) using polystyrene as standard. Synthesis of poly[(9,9-bis\{propenyl\}fluorenyl-2,7-diyl)-co-(9,9-dihexylfluorenyl-2,7-diyl)] (P1).

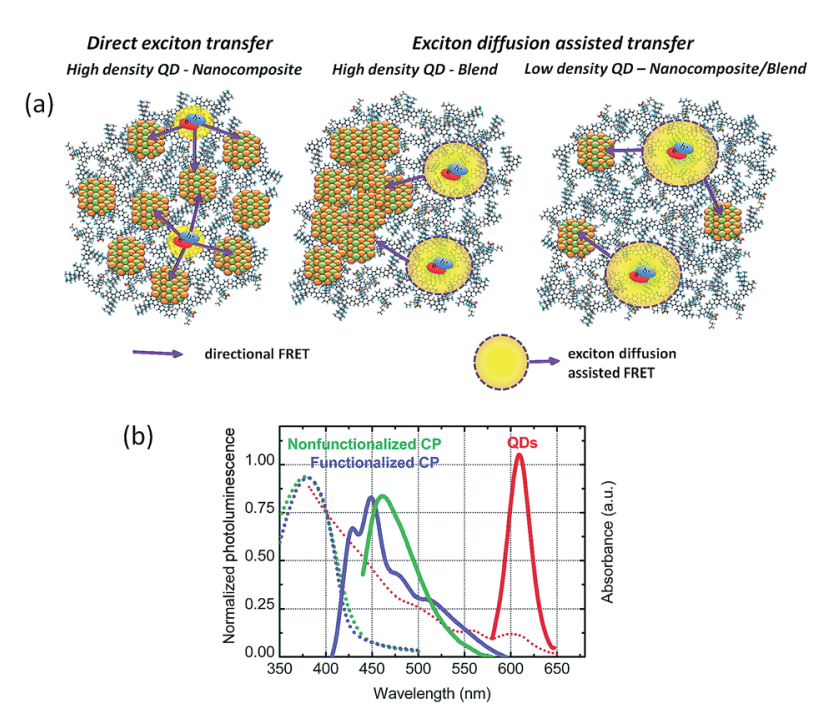

Fig. 1 (a) Representative schematic for the exciton transfer in the high density QD incorporated (left) nanocomposite, (middle) blend and the low density QD incorporated (right) nanocomposite and blend. (b) Normalized photoluminescence (solid) and absorbance (dotted) spectra of the functionalized CP, nonfunctionalized CP and the QDs.
2,7-Dibromo-9,9-bis-(propenyl)-9H-fluorene (1.00 g, 2.474 mmol), 9,9-dihexylfluorene-2,7-bis(trimethyleneborate) (1.243 g, $2.474 \mathrm{mmol})$ and $\mathrm{K}_{2} \mathrm{CO}_{3}(3.419 \mathrm{~g}, 24.74 \mathrm{mmol})$ were dried under vacuum while THF, $\mathrm{H}_{2} \mathrm{O}$ and toluene were degassed under argon for about $15 \mathrm{~min}$. Then the mixture was suspended into degassed THF $(10 \mathrm{~mL})$, water $(10 \mathrm{~mL})$ and toluene $(10 \mathrm{~mL})$ and catalyst tetrakis(triphenylphosphine) palladium $(0)\left(\mathrm{Pd}\left(\mathrm{PPh}_{3}\right)_{4}\right)(0.0286 \mathrm{~g}$, $0.025 \mathrm{mmol}$ ) was added quickly. After $3 \mathrm{~h}$ phase transfer catalyst tetra- $n$-butylammonium bromide (TBAB) $(0.0079 \mathrm{~g}, 0.025 \mathrm{mmol})$ was added and the resulting mixture was stirred under argon gas for $48 \mathrm{~h}$ at $80-90{ }^{\circ} \mathrm{C}$. At the end of the reaction, the solvent was evaporated to obtain a solid residue which was suspended in a chloroform-water mixture. The organic layer was separated and evaporated to the reduced volume and precipitated into cold methanol $(100 \mathrm{~mL})$. The precipitates were collected by suction filtration; washed with water several times and redissolved in a minimum amount of THF. The solution was reprecipitated into excess cold methanol. Light yellow colored precipitates were collected by filtration and dried under vacuum for $6 \mathrm{~h}$.

Yield: 93\%. 1H-NMR (400 MHz, $\left.\mathrm{CDCl}_{3}, 25{ }^{\circ} \mathrm{C}\right), \delta \mathrm{H} 7.85(\mathrm{~m}$, $8 \mathrm{H}, \mathrm{Ar} \mathrm{H}), 5.54$ (q, 2H, CH), $5.02\left(\mathrm{t}, 4 \mathrm{H}, \mathrm{CH}_{2}\right), 2.89\left(\mathrm{~m}, 4 \mathrm{H}, \mathrm{CH}_{2}\right)$, $1.54\left(\mathrm{q}, 4 \mathrm{H}, \mathrm{CH}_{2}\right), 1.15\left(\mathrm{~m}, 4 \mathrm{H}, \mathrm{CH}_{2}\right), 0.81\left(\mathrm{~m}, 6 \mathrm{H}, \mathrm{CH}_{3}\right)$.

GPC: $M_{\mathrm{n}}=7.01 \times 10^{3} M_{\mathrm{w}}=9.02 \times 10^{3}$ (polystyrene as standard).

Synthesis of poly[(9,9-bis\{carboxymethylsulfonyl-propyl\}fluorenyl-2,7-diyl)-co-(9,9-dihexylfluorenyl-2,7-diyl)] (functionalized CP). Poly[(9,9-bis\{propenyl\}fluorenyl-2,7-diyl)-co-(9,9dihexylfluorenyl-2,7-diyl)] (P1) was dissolved in $\mathrm{CHCl}_{3}$ and excess mercaptoacetic acid was added. The mixture was stirred under Ar for $24 \mathrm{~h}$ at room temperature. Solvent was removed under reduced pressure and dissolved in THF and the resulting solution was precipitated into water to yield a light yellow color functionalized CP, which was dried under vacuum for $6 \mathrm{~h}$.

\section{Synthesis of the core-shell QDs}

For the growth of CdSe cores, the synthesis started with mixing $64 \mathrm{mg}$ of cadmium oxide (CdO), $183.5 \mathrm{mg}$ of zinc acetate (ZnAc) and $2.5 \mathrm{~mL}$ of oleic acid (OA). In an air free environment by using vacuum conditions, the mixture was heated up to $150{ }^{\circ} \mathrm{C}$ and kept at this temperature for 30 minutes. Then, the system was taken from vacuum to argon flow and $12.5 \mathrm{~mL}$ of degassed 1-octadecene (ODE) was added quickly into the prepared mixture. After addition of ODE, the temperature of the system was increased to $300{ }^{\circ} \mathrm{C}$. At the reported elevated temperature, $0.1 \mathrm{~mL}$ of injection solution of selenium (Se), which was prepared as $1 \mathrm{M}$ in trioctylphosphine (TOP), was injected quickly into the clear reaction mixture, and the core growth continued for $2.5 \mathrm{~min}$. Subsequently, $0.15 \mathrm{~mL}$ of dodecanethiol

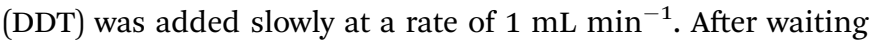
for $20 \mathrm{~min}$ for the formation of CdZnS layer overcoating the cores, $0.5 \mathrm{~mL}$ of injection solution of sulphur (S), prepared as 2 $\mathrm{M}$ in TOP, was added into the reaction mixture quickly. For the formation of the final $\mathrm{ZnS}$ shell, $10 \mathrm{~min}$ growth time was used. At the end of the synthesis, the solution was cooled down to room temperature to stop the growth. These QDs in solution have photoluminescence quantum efficiencies up to $50 \%$. 


\section{Hybrid film preparation}

$20 \mathrm{mg} \mathrm{mL} \mathrm{m}^{-1}$ of functionalized $\mathrm{CP}$ in THF and $10 \mathrm{mg} \mathrm{mL}^{-1}$ of core-shell QDs in toluene were mixed in the desired ratio. The mixture was left on a vortex overnight. Subsequently, the mixture was spin-coated using $2000 \mathrm{rpm}$ for $1.5 \mathrm{~min}$. The resulting thin film thickness was measured to be $60 \mathrm{~nm}$ using a profilometer.

\section{SEM and TEM characterization}

Scanning electron microscopy (Quanta 200 FEG, FEI) of the nanocomposite and blend films spin-coated on (100) p-doped silicon substrates was carried out. An energy dispersive X-ray spectrometer integrated with a SEM system (EDAX, Materials Analysis Division) was used to understand the phase segregated parts of the blended films. Transmission electron microscopy (Technai G2 F30, FEI) was used with ultra thin carbon grids in scanning mode via a high sensitivity HAADF STEM detector.

\section{Time resolved fluorescence spectroscopy}

A time resolved fluorescence spectrometer (Fluotime 200) with a time correlated single photon counting system (TCSPC) (Pico Harp 300) integrated with a closed cycle He cryostat was used for measuring the fluorescence decays. A picosecond pulsed laser working at $375 \mathrm{~nm}$ operating at $5 \mathrm{MHz}$ repetition rate with an average optical power level of $0.5 \mathrm{~mW} \mathrm{~cm}^{-2}$ was utilized. The instrument response function of the system has a full width at half maximum around $200 \mathrm{ps}$.

\section{Device fabrication}

The device fabrication started with the substrate preparation. We used square glass/ITO substrates with $15 \Omega \mathrm{sq}^{-1}$ sheet resistance (Kintec) of $1.5 \mathrm{~cm} \times 1.5 \mathrm{~cm}$ in size. First, we etched the side of the ITO using acidic etchant mixture $\mathrm{HCl}: \mathrm{HNO}_{3}$ $: \mathrm{H}_{2} \mathrm{O}(4.6: 0.4: 5)$. Then, we cleaned substrates using solvent cleaning (deionized water-detergent (Hellmanex III, Hellma Analytics) mixture, deionized water, acetone, and isopropanol for $15 \mathrm{~min}$ each). After the solvent cleaning, substrates were UV-ozone treated right before spin-coating PEDOT:PSS (using 500,4000 and $5000 \mathrm{rpm}$ for 5,120 and $120 \mathrm{~s}$, respectively). Then, the films were annealed in a glove box at $140{ }^{\circ} \mathrm{C}$ for 2 hours. After, a mixture of hybrid solution was prepared and mixed with a vortex for a few hours. The hybrid solution was spin-coated at $2000 \mathrm{rpm}$ for $2 \mathrm{~min}$. The active layer was annealed at $70^{\circ} \mathrm{C}$. In a thermal evaporator, a $100 \mathrm{~nm}$ thick $\mathrm{Al}$ film was deposited. Later, devices were encapsulated with a cover glass using epoxy.

\section{Results and discussion}

In the CP-QD systems phase segregation causes QDs to form aggregates in the CP host, and therefore suppresses FRET from the CP to the QDs since the interaction between the donor and acceptor is considerably limited. For this reason, phase segregation should be minimized for efficient energy transfer pumping to the QDs from the CPs. There were several reports on developing chemically integrated $\mathrm{CP}-\mathrm{QD}$ nanocomposites. ${ }^{10,22,23}$ Among these, grafting the CPs to the QD surface via active moieties of the side-chains of the CPs is a versatile method without impairing the original photophysical properties of the QDs. ${ }^{23}$ Previously, such grafted CP-QD composites were used for hybrid solar cells ${ }^{24}$ or light-emitting diodes. ${ }^{25-27}$ However, these nanocomposites were not specifically studied for their spectroscopic properties, nor exciton transfer was understood. In this work, a functionalized CP (poly[(9,9-bis\{carboxymethylsulfonyl-propyl\}fluorenyl-2,7-diyl)-co(9,9-dihexylfluorenyl-2,7-diyl)]) is synthesized (see Fig. S1 $\dagger$ ), which has carboxymethylsulfonyl functionalized side groups to interact specifically with the QD surfaces. Nonfunctionalized CP (poly[(9,9-dioctylfluorenyl-2,7-diyl)-co-( $N, N^{\prime}$-diphenyl)- $N, N^{\prime}$-di( $p$-butyloxy-phenyl)-1,4-(diaminobenzene)] - American dye source 232 $\mathrm{GE}$ ) has a similar backbone compared to the functionalized CP, yet it does not have any specific active side-chain moieties. As the QDs, we synthesize red-emitting core-shell CdSe/ZnS QDs having an average diameter of $6.3 \mathrm{~nm}$ (see Fig. $\mathrm{S} 2 \uparrow$ for the TEM images). ${ }^{28}$ Fig. 1b depicts the fluorescence emission and absorbance of the functionalized and nonfunctionalized CPs and the QDs.

\section{Suppression of the phase segregation}

Thin films of the functionalized CP-QD nanocomposites and the nonfunctionalized CP-QD blends were fabricated via spin coating over cleaned quartz substrates. Two different QD concentrations were used: low density (QD : CP $=3$ wt $\%: 97$ wt $\%$ ) and high density (QD : CP $=45 \mathrm{wt} \%: 55 \mathrm{wt} \%$ ). For the case of high density QDs in the functionalized CP the scanning electron microscopy (SEM) image in Fig. 2a does not exhibit any indication of phase segregation at a resolution level of a few hundred nanometers, whereas Fig. 2c depicts the SEM image of the blend with high density QDs, where severe phase segregation is clearly visible. Fig. $2 \mathrm{~b}$ shows the high-angle annular darkfield scanning transmission electron microscopy (HAADF STEM) image of the high density QD loaded nanocomposite that reveals suppressed phase segregation owing to the presence of the active carboxyl acid moieties at the side chains of the functionalized CP, which act as multi-ligands for the QDs (see $\mathrm{ESI} \dagger$ for the XPS analysis that reveals the strong interaction between the side chains of the functionalized CP and the QD surface). In addition, we always observe amorphous cloud around the QDs in HAADF-STEM images having darker color than the carbon grid background, which is possibly attributed to the surrounding functionalized CPs around the QDs. However, this darker colored cloud is never observed for the same QDs when mixed with the nonfunctionalized CP (see Fig. 2d).

\section{Temperature dependent energy transfer dynamics}

The time correlated single photon counting (TCSPC) technique is employed to measure the fluorescence decay kinetics of the donor CPs. In the absence of QDs, the fluorescence decays of the CPs is fitted well using the single exponential decay function with a near-unity reduced $\chi^{2}$ after deconvoluting with the instrument response function. To compute the FRET efficiencies $(\eta)$ in the case of the blends, we employ the fitted amplitude 
(a)

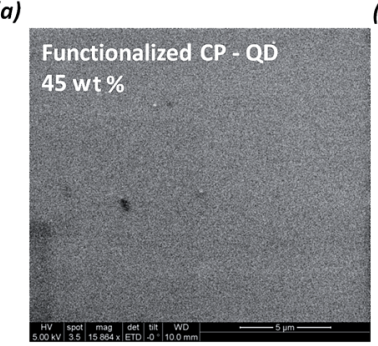

(c)

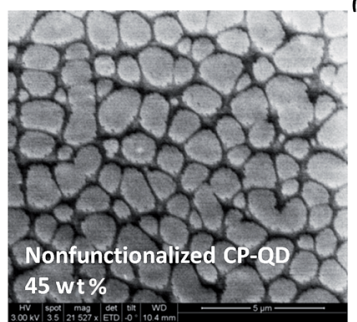

(b)

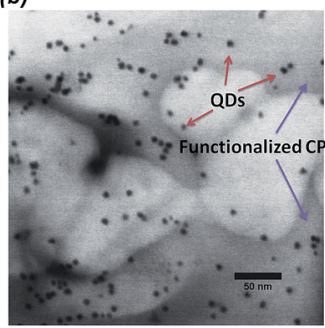

(d)

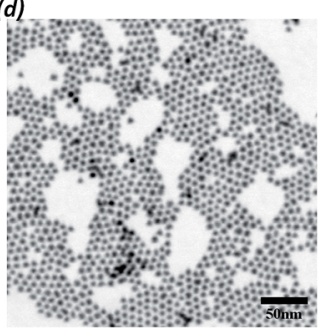

Fig. 2 Scanning electron microscopy (SEM) of (a) the functionalized CP-QD nanocomposite and (c) the nonfunctionalized CP-QD blend with the same QD density of 45 wt\%. High-angle annular dark-field scanning transmission electron microscopy (HAADF STEM) images of (b) the functionalized CP-QD nanocomposite and (d) the nonfunctionalized CP-QD blend with the same QD density of 45 wt\%.

averaged fluorescence lifetimes of the CP in the absence $\left(\tau_{\mathrm{CP}}\right)$ and presence $\left(\tau_{\mathrm{CP}-\mathrm{QD}}\right)$ of the QDs $\left(\eta=1-\frac{\tau_{\mathrm{CP}-\mathrm{QD}}}{\tau_{\mathrm{CP}}}\right)$. For the nanocomposites, we apply the Loring-Anderson-Fayer (LAF) approach to analyze the fluorescence decays. ${ }^{20,29}$ The LAF method extends decay kinetics described by the Förster theory for multi-acceptors in the three-dimension. ${ }^{30} \mathrm{ESI} \dagger$ describes the LAF approach in more detail. The FRET efficiencies are calculated using eqn (S8). $\uparrow$ Fig. $3 \mathrm{a}$ and b plot the FRET efficiencies as a function of temperature for the nanocomposite and the blend at $3 \mathrm{wt} \%$ and $45 \mathrm{wt} \%$ density QD loadings. Low density QD incorporation into both CPs leads to high-temperature sensitive FRET kinetics. This observation is in accordance with the essentially exciton diffusion assisted FRET process. ${ }^{20}$ Exciton diffusion in a CP is suppressed at low temperatures due to the absence of thermal activation energy; ${ }^{31}$ therefore, FRET from the CP to the QDs is consequently suppressed ( $<150 \mathrm{~K})$. Even negative FRET efficiencies are calculated in the blend. We attribute the negative FRET efficiencies at the cryogenic
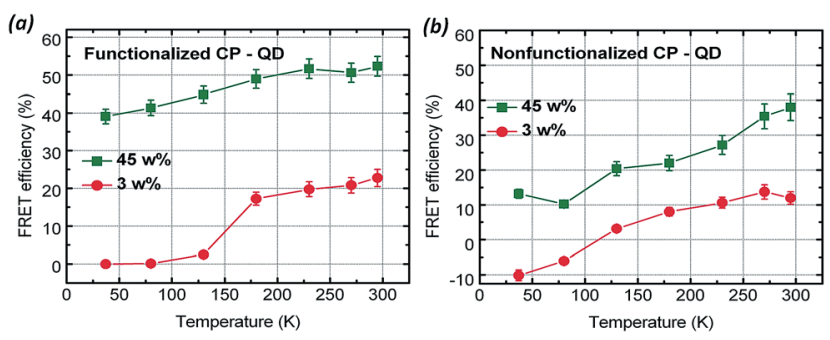

Fig. 3 Temperature dependent FRET efficiencies are represented for the 3 and 45 wt\% density QD incorporated (a) nanocomposite and (b) blend. temperatures to the possible morphological phase change of the nonfunctionalized CP at low temperatures, which alters the excited state relaxation dynamics of the CP. Also, this phase change may take place differently in the presence of the QDs leading to the calculation of negative FRET efficiencies as also observed previously. ${ }^{19,20,32}$

A fundamental difference in temperature-dependent FRET kinetics is observed in the case of high density QD loadings. In the nanocomposite with high density QDs, FRET efficiencies are observed to exhibit much weaker temperature sensitivity (see Fig. 3a). More than $80 \%$ of the FRET efficiency at room temperature is sustained even at the lowest temperature case of $37 \mathrm{~K}$. This affirms that exciton diffusion does not critically assist FRET in the nanocomposite with high density QDs. Thus, direct exciton transfer from the functionalized CP to the QDs is viable. Nonetheless, in the blend at high density QDs, the temperature sensitivity of FRET is still high similar to its low density QD loaded counterpart (see Fig. 3b). At the lowest temperature, less than $30 \%$ of the room temperature FRET efficiency could be sustained in the blend with high density QDs. This is due to the fact that aggregation of the QDs in the blend inhibits the direct exciton transfer and exciton diffusion assistance is still required.

\section{Energy transfer models: exciton transfer vs. exciton diffusion}

We model the exciton transfer and the exciton diffusion dynamics in the nanocomposites using the Gösele approach. ${ }^{33}$ Contribution from the exciton diffusion is explicitly included in the Gösele model to account for the exciton diffusion assistance to FRET (see ESI† for more details about the Gösele model). Here, we do not consider the blend cases since it is difficult to consider the effects of random aggregates that are observed in the blends at high density QD loadings. Eqn (S9)† relates the donor fluorescence lifetime that is modified due to the presence of acceptors. In this equation, diffusion coefficient $(D$, in units of $\mathrm{nm}^{2} \mathrm{~ns}^{-1}$ ) is the only unknown parameter. The density of the acceptors $\left(n_{\mathrm{A}}\right.$, in units of \#QDs $\left.\mathrm{nm}^{-3}\right)$ is estimated in the nanocomposites via measuring the absorbance of the QDs in the hybrid films and comparing them with the known concentration solutions of the QDs. $n_{\mathrm{A}}$ is $\sim 4.0 \times 10^{-5} \mathrm{QDs} \mathrm{nm}^{-3}$ and $\sim 3.6 \times 10^{-4}$ QDs $\mathrm{nm}^{-3}$ for the cases of $3 \mathrm{wt} \%$ and $45 \mathrm{wt} \%$ density QDs, respectively. Then, we model the donor fluorescence lifetime of the functionalized CP using eqn (S9) $\dagger$ parameterized with respect to $D$ and $n_{\mathrm{A}}$. In the ESI $\dagger$ we present the analysis to cross-check the Gösele approach by calculating the time expected value of $\tau_{\mathrm{DA}}$ using eqn (S12) $\dagger$ as shown in Fig. $\mathrm{S} 4, \dagger$ where time expected values of the modeled donor fluorescence lifetimes match very well with the experimental donor fluorescence lifetimes. Fig. $4 \mathrm{a}$ and $\mathrm{b}$ plot the iso-fluorescence lifetime (iso- $\tau_{\mathrm{DA}}$ ) of the donor, i.e., the functionalized $\mathrm{CP}$, in the presence of acceptors at two exemplary temperature points of $295 \mathrm{~K}$ and $180 \mathrm{~K}$, respectively. Iso-fluorescence lifetimes are shown for the rest of the temperature points in Fig. S5. $\dagger$ In Fig. $4 \mathrm{a}$ and b, modeled iso- $\tau_{\mathrm{DA}}$ curves are plotted with varying color shades, where $\tau_{\mathrm{DA}}$ becomes longer as the shades vary from light brown to darker brown color. It is 

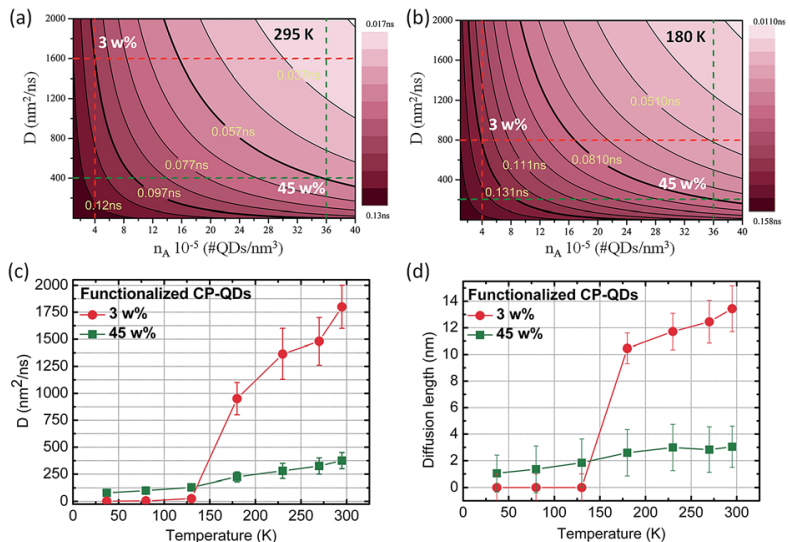

Fig. 4 Iso- $\tau_{\mathrm{DA}}$ curves, modeled by the Gösele approach, are plotted as a function of $D$ and $n_{\mathrm{A}}$ at (a) 295 and (b) $180 \mathrm{~K}$. Diffusion coefficient, $D$, which matches the modeled $-\tau_{D A}$ to the experimental $-\tau_{D A}$, is highlighted by red and green dashed lines for 3 wt $\%$ and 45 wt\% QD loading, respectively. (c) $D$ as a function of temperature for the 3 and $45 w t \%$ density QD loaded nanocomposites. (d) Diffusion length $\left(L_{D}\right)$ as a function of temperature for the cases of 3 and 45 wt\% QD loaded nanocomposites.

observed that increasing either $n_{\mathrm{A}}$ and $D$ favors a decrease of $\tau_{\mathrm{DA}}$ since increase in these parameters (i.e., $D$ and $n_{\mathrm{A}}$ ) favors an increased FRET rate from the CP to the QD. In each Fig. 4a and $\mathrm{b}$, two iso- $\tau_{\mathrm{DA}}$ curves are made bold, which represent the experimental $\tau_{\mathrm{DA}}$ (i.e., $0.097 \mathrm{~ns}$ and $0.057 \mathrm{~ns}$ in Fig. 4a for $45 \mathrm{wt} \%$ and $3 \mathrm{wt} \%$ density QD loaded nanocomposite, respectively). We mark the $D$ values by horizontal dashed lines (red color for $3 \mathrm{wt} \%$ and green color for $45 \mathrm{wt} \%$ ), which match the modeled- $\tau_{\mathrm{DA}}$ to the experimental $\tau_{\mathrm{DA}}$ for the given $n_{\mathrm{A}}$ at the low or high density QD incorporated nanocomposites.

Fig. 4c shows the diffusion coefficients $(D)$ that are extracted by matching the modeled iso- $\tau_{\mathrm{DA}}$ to the experimental $\tau_{\mathrm{DA}}$ using the Gösele model. Here, we observe a remarkable difference in the exciton diffusion characteristics of the functionalized CP depending on the density of the QD loading. At room temperature, $D$ is found to be $1800 \mathrm{~nm}^{2} \mathrm{~ns}^{-1}$ for the $3 \mathrm{wt} \%$ QD loaded nanocomposite, whereas $D$ is only $350 \mathrm{~nm}^{2} \mathrm{~ns}^{-1}$ for the $45 \mathrm{wt} \%$ QD loaded nanocomposite. The higher diffusion coefficient in the low density QD loaded nanocomposite is in agreement with the $D$ of a similar type of polyfluorene derivative polymer, which was found to have $1440 \pm 250 \mathrm{~nm}^{2} \mathrm{~ns}^{-1}$ at room temperature. ${ }^{34}$ This indicates that intrinsic exciton diffusion properties of the functionalized CP are preserved in the case of low density QDs in the nanocomposite. However, in the high density QD incorporated nanocomposite, $D$ is observed to be much smaller as compared to $D$ in the case of low density QDs. This indicates that exciton diffusion is slowed down due to the incorporation of a larger amount of QDs into the nanocomposite. As shown in Fig. $4 \mathrm{c}, D$ is found to be decreased by 5 -fold in the $45 \mathrm{wt} \%$ nanocomposite as compared to the $3 \mathrm{wt} \%$ nanocomposite. One possibility to explain this result is to refer to the two fundamental mechanisms of exciton diffusion in the CPs, so-called inter- and intra-chain exciton diffusion. The inter-chain diffusion has been shown to be a much faster process than the intra-chain diffusion, and the inter-chain diffusion was claimed to be dominant in highly stacked solidstate films of the CPs due to close packing of the polymer chains. $^{35,36}$ On the other hand, the intra-chain diffusion becomes dominant when the $\mathrm{CP}$ chains are more isolated as in the case of dilute solutions. Thus, the decrease of diffusion coefficient at high QD densities can be possibly attributed to the morphological changes in the polymer matrix. The CP chains are more isolated from each other in the case of high density nanocomposites as compared to the low density case. Hence, slower intra-chain diffusion may dominate as opposed to faster inter-chain diffusion.

The temperature dependent change of $D$ in low and high density QD cases is also different. As the temperature is reduced from room temperature down to $150 \mathrm{~K}, D$ is reduced substantially in the case of low QD density nanocomposites. There is a turn-off temperature below $150 \mathrm{~K}$ as observed before. $^{20}$ However, in the case of high density nanocomposites there is a monotonic decrease of $D$ as the temperature is decreased. Exciton diffusion in the CPs is a temperature activated process that takes place via hopping of the excitons over different units of the polymer chains, where extra energy (i.e., $k T$ ) is required to jump over the low energy traps in the disordered density of states (DOS) of the CP. ${ }^{35}$ Therefore, decrease of $D$ as the temperature is reduced is expected. Yet, behavior below $150 \mathrm{~K}$ may be attributed to changing conformation and inter-chain packing of the polymer at the cryogenic temperatures, which are observed only in the low density QD incorporated nanocomposites. In the case of high QD loading, exciton diffusion is highly slowed down.

In Fig. $4 \mathrm{~d}$ we plot the exciton diffusion length $\left(L_{\mathrm{D}}=\sqrt{D \tau_{\mathrm{D}}}\right)$ in the nanocomposites of low and high density QDs using the estimated diffusion coefficient $D$ (Fig. 4c) and the experimentally known fluorescence lifetime of the CP. $L_{\mathrm{D}}$ also exhibits a significant difference between the low and the high density nanocomposites. For the low density, $L_{\mathrm{D}}$ is found to be up to 13 $\mathrm{nm}$ at room temperature, whereas for the high density case $L_{\mathrm{D}}$ is only up to $3 \mathrm{~nm}$. Thus, excitons can on average diffuse up to 4-fold longer distances in the case of the low density nanocomposite as compared to the high density one. The exciton diffusion length that is slightly larger than $10 \mathrm{~nm}$ is in agreement with the previous literature for similar types of polymers. ${ }^{37}$ This indicates that a small amount of QD incorporation into the nanocomposite does not severely disturb the exciton diffusion properties of the CP. However, in the case of high density QDs, exciton diffusion length is very limited (1-3 nm); thus, exciton diffusion is suppressed in the high density QD incorporated nanocomposite. This suppressed exciton diffusion is in accordance with only-downhill exciton diffusion in the CPs, where downhill relaxation of the exciton in the disordered density of states of the CPs is estimated to be $2.0-2.5 \mathrm{~nm} .{ }^{37}$ Therefore, for the high density QD incorporated nanocomposite, excitons are transferred to the QDs after only-downhill diffusion of the energetic excitons in the disordered DoS of the CP. We present steady state fluorescence measurements of the nanocomposites 
as a function of temperature as an additional proof of the change of the exciton diffusion properties with QD loading in the nanocomposite in Fig. $56, \uparrow$ which reveals the spectroscopic signature of the exciton diffusion suppression in the CP emission at high density QD loading.

\section{Exciton transfer efficiency vs. QD loading}

To reveal the potential of these functionalized CP-QD nanocomposites as an efficient exciton transferring media, we further study FRET efficiencies in the nanocomposites as a function of varying densities of QDs at room temperature as shown in Fig. 5a. FRET efficiencies can be as high as $80 \%$ when the density of QDs is as high as $70 \mathrm{wt} \%$. Here, steady state photoluminescence of the $70 \mathrm{wt} \%$ density QD loaded nanocomposite is also plotted with its control group samples (bare donor and acceptor) in Fig. 5b, where quenching of the CP fluorescence emission and concomitant enhancement of the QD fluorescence emission are clearly visible. The fluorescence emission of the QDs is red-shifted in the nanocomposite as attributed to the better spectral overlap of the slightly larger QDs in the inhomogeneously broadened size distribution. Another proof for this efficient exciton transfer is shown in Fig. S3, $\uparrow$ where fluorescence decay of the QDs in the nanocomposite and the bare QDs is plotted. The fluorescence lifetime of the QDs increases when QDs are in the nanocomposite as compared to the fluorescence decay of the bare QDs. This can be explained by the exciton feeding from the CP donor. ${ }^{12,38-40}$ Also, better surface passivation of the QDs within the nanocomposite might have helped. Moreover, charge transfer from the CP to the QDs is not possible in our system due to the thick $\mathrm{ZnS}$ shell ( $>3$ monolayers) of the QDs. ${ }^{41}$ Therefore, inter-QD FRET ${ }^{42}$ or inter-QD exciton coupling ${ }^{43}$ is expected to be quite weak in our case. Previously, Lutich et al. have not even observed significant charge transfer from a similar type of CP to core only CdTe QDs. ${ }^{17}$

\section{Exciton transfer pumped hybrid LEDs}

Finally, a proof-of-concept demonstration of the CP-QD system as a light-emitting medium for light-emitting diodes is presented. Devices are fabricated using a simple device (a)

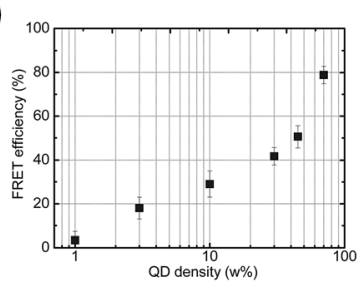

(b)

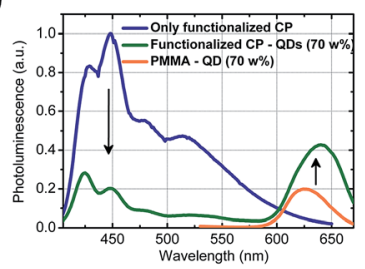

Fig. 5 (a) FRET efficiencies are measured as a function of QD density in the hybrid nanocomposite at room temperature. QD densities of $3 \mathrm{wt} \%$ and $45 \mathrm{wt} \%$ correspond to average QD-to-QD distance of 16 and $7 \mathrm{~nm}$ in their nanocomposites, respectively. (b) Steady state photoluminescence of only functionalized $C P$, functionalized $C P-Q D$ (70 wt\%) and PMMA-QD (70 wt\%). architecture consisting of ITO/PEDOT:PSS/emissive layer/Al, where the emissive layer consisted of either the bare CPs, the bare QDs, the nanocomposites or the blends. For the nanocomposite-based LEDs, i.e., nanocomposite-LEDs, high density QDs up to $70 \mathrm{wt} \%$ are employed to facilitate efficient FRET for a strong energy transfer pumping to the QDs from the functionalized CP. In the case of blend-LEDs, strong energy transfer pumping could never be achieved since phase segregation is inevitable. The bare QD-LEDs (without any polymer host) exhibited weak electroluminescence (EL) due to the poor electrical injection in the simple device architecture employed here. On the other hand, nanocompositeLEDs demonstrated highly stable and enhanced EL spectra with a dominant QD emission as shown in Fig. 6a. In these nanocomposite-LEDs, emission of the functionalized CP is substantially suppressed owing to the efficient exciton transfer into the QDs. Although we have previously measured $80 \%$ FRET efficiency in the $70 \mathrm{wt} \%$ density QD loaded nanocomposite (see Fig. 5a), the observation of almost totally suppressed emission from the CP could be also due to the change of the exciton transfer pathways in the electroluminescent devices. In contrast, blend-LEDs always exhibit mixed emission both from the QDs and the nonfunctionalized CP in their EL spectra (Fig. 6a) due to limited and incomplete energy transfer pumping in these phase separated structures. In terms of the external quantum efficiencies (EQE) of the devices, nanocompositeLEDs enable a significant enhancement, which is more than an order of magnitude, in EQEs as compared to bare QD-LEDs and bare functionalized CP-LEDs as depicted in Fig. 6b. Bare QD-LEDs suffer considerably from the poor charge injection and bare functionalized CP-LEDs suffer from the defects that are highly populated due to the exciton diffusion. ${ }^{\mathbf{4 4}}$ Therefore, in the nanocomposite-LEDs, excitation energy in the CP could be much more effectively utilized through transferring them to the QDs resulting in substantially enhanced EQEs. With careful design of the device architecture, we believe that these excitonic nanocomposites will be promising for high efficiency hybrid LEDs in future. (a)

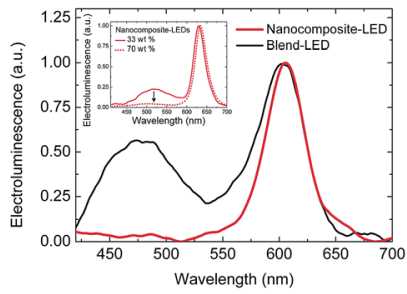

(b)

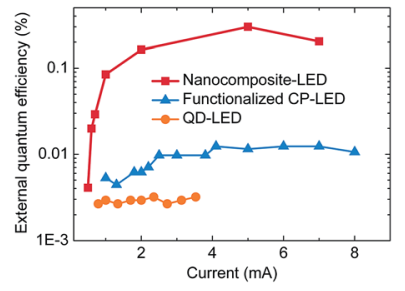

Fig. 6 (a) Normalized electroluminescence spectra of the nanocomposite-LED and blend-LED having $70 \mathrm{wt} \%$ density QDs. The inset shows the normalized electroluminescence spectra of the nanocomposite-LEDs with two different QD densities of 33 wt $\%$ and $70 \mathrm{wt} \%$. (b) External quantum efficiency of the nanocomposite-LED, bare functionalized CP-LED and bare QD-LED. 


\section{Conclusion}

In conclusion, we study and reveal the excitonic interactions in the conjugated polymer-quantum dot hybrid nanocomposites and blends at low and high density QDs. For such an exciton transfer process, it was believed that exciton diffusion crucially assists FRET. Here, we reveal the unknown territory of high density QD incorporated nanocomposites. With the help of the side-chain functionalized $\mathrm{CP}$, we achieve phase segregation suppressed nanocomposite hybrid films, which show highly efficient exciton transfer (as high as 80\%) even at low temperatures. In these high density QD incorporated nanocomposites exciton diffusion assistance to exciton transfer is not required. Instead, direct exciton transfer channel prevails. We employed energy transfer models to explain the interplay between the exciton transfer and the exciton diffusion. At high QD densities, the exciton diffusion coefficient is shown to be suppressed by 5 -fold. This new understanding and finding is applied to design efficient energy transfer pumped hybrid LEDs that show an order of magnitude enhanced performance.

\section{Acknowledgements}

This work is supported in part by EU-FP7 Nanophotonics4Energy NoE, and TUBITAK EEEAG 109E002, 109E004, 110E010, and 110E217, in part by NRF-CRP-6-2010-02 and NRFRF-2009-09. H. V. D. acknowledges support from ESF-EURYI and TUBA-GEBIP; A. O. G. was supported by U.S. Army Research Office (USA) under grant number W911NF-12-1-0407.

\section{References}

1 Y. Shirasaki, G. J. Supran, M. G. Bawendi and V. Bulović, Nat. Photonics, 2013, 7, 933.

2 E. H. Sargent, Nat. Photonics, 2012, 6, 133-135.

3 G. Konstantatos, M. Badioli, L. Gaudreau, J. Osmond, M. Bernechea, F. P. Garcia de Arquer, F. Gatti and F. H. L. Koppens, Nat. Nanotechnol., 2012, 7, 363-368.

4 C. Dang, J. Lee, C. Breen, J. S. Steckel, S. Coe-Sullivan and A. Nurmikko, Nat. Nanotechnol., 2012, 7, 335-339.

5 J. H. Burroughes, D. D. C. Bradley, A. R. Brown, R. N. Marks, K. Mackay, R. H. Friend, P. L. Burns and A. B. Holmes, Nature, 1990, 347, 539-541.

6 N. S. Sariciftci, L. Smilowitz, A. J. Heeger and F. Wudl, Science, 1992, 258, 1474-1476.

7 H. Koezuka, A. Tsumura and T. Ando, Synth. Met., 1987, 18, 699-704.

8 W. U. Huynh, J. J. Dittmer and A. P. Alivisatos, Science, 2002, 295, 2425-2427.

9 V. L. Colvin, M. C. Schlamp and A. P. Alivisatos, Nature, 1994, 370, 354-357.

10 P. Reiss, E. Couderc, J. De Girolamo and A. Pron, Nanoscale, 2011, 3, 446-489.

11 N. Tessler, V. Medvedev, M. Kazes, S. Kan and U. Banin, Science, 2002, 295, 1506-1508.
12 B. Guzelturk, P. L. H. Martinez, Q. Zhang, Q. Xiong, H. Sun, X. W. Sun, A. O. Govorov and H. V. Demir, Laser Photonics Rev., 2014, 8, 73-93.

13 M. Anni, L. Manna, R. Cingolani, D. Valerini, A. Cretí and M. Lomascolo, Appl. Phys. Lett., 2004, 85, 4169.

14 T.-W. F. Chang, S. Musikhin, L. Bakueva, L. Levina, M. A. Hines, P. W. Cyr and E. H. Sargent, Appl. Phys. Lett., 2004, 84, 4295.

15 P. T. K. Chin, R. A. M. Hikmet and R. A. J. Janssen, J. Appl. Phys., 2008, 104, 013108.

16 S. Kaufmann, T. Stoferle, N. Moll, R. F. Mahrt, U. Scherf, A. Tsami, D. V. Talapin and C. B. Murray, Appl. Phys. Lett., 2007, 90, 071108.

17 A. A. Lutich, G. Jiang, A. S. Susha, A. L. Rogach, F. D. Stefani and J. Feldmann, Nano Lett., 2009, 9, 2636-2640.

18 J. H. Warner, A. R. Watt, E. Thomsen, N. Heckenberg, P. Meredith and H. Rubinsztein-Dunlop, J. Phys. Chem. B, 2005, 109, 9001-9005.

19 A. A. Lutich, A. Poschl, G. Jiang, F. D. Stefani, A. S. Susha, A. L. Rogach and J. Feldmann, Appl. Phys. Lett., 2010, 96, 083109.

20 T. Stoferle, U. Scherf and R. F. Mahrt, Nano Lett., 2009, 9, 453-456.

21 N. Greenham, X. Peng and A. Alivisatos, Phys. Rev. B: Condens. Matter Mater. Phys., 1996, 54, 17628-17637.

22 N. Tomczak, D. Jańczewski, M. Han and G. J. Vancso, Prog. Polym. Sci., 2009, 34, 393-430.

23 N. Tomczak, R. Liu and J. G. Vancso, Nanoscale, 2013, 5, 12018-12032.

24 D. J. Milliron, A. P. Alivisatos, C. Pitois, C. Edder and J. M. J. Fréchet, Adv. Mater., 2003, 15, 58-61.

25 H. Skaff, K. Sill and T. Emrick, J. Am. Chem. Soc., 2004, 126, 11322-11325.

26 J. Kwak, W. K. Bae, M. Zorn, H. Woo, H. Yoon, J. Lim, S. W. Kang, S. Weber, H.-J. Butt, R. Zentel, S. Lee, K. Char and C. Lee, Adv. Mater., 2009, 21, 5022-5026.

27 M. Zorn, W. K. Bae, J. Kwak, H. Lee, C. Lee, R. Zentel and K. Char, ACS Nano, 2009, 3, 1063-1068.

28 W. K. Bae, K. Char, H. Hur and S. Lee, Chem. Mater., 2008, 20, 531-539.

29 R. Loring, H. Andersen and M. Fayer, J. Chem. Phys., 1982, 76, 2015-2027.

30 J. R. Lakowicz, Principles of Fluorescence Spectroscopy, 2007.

31 F. B. Dias, K. T. Kamtekar, T. Cazati, G. Williams, M. R. Bryce and A. P. Monkman, ChemPhysChem, 2009, 10, 2096-2104.

32 X. Zhang, Z. Li and G. Lu, Phys. Rev. B: Condens. Matter Mater. Phys., 2011, 84, 235208.

33 U. Gösele, M. Hauser, U. Klein and R. Frey, Chem. Phys. Lett., 1975, 34, 519-522.

34 U. Scherf and D. Neher, Polyfluorenes, Springer, 2008, 212.

35 D. Beljonne, G. Pourtois, C. Silva, E. Hennebicq, L. M. Herz, R. H. Friend, G. D. Scholes, S. Setayesh, K. Mullen and J. L. Bredas, Proc. Natl. Acad. Sci. U. S. A., 2002, 99, 1098210987.

36 V. Gulbinas, I. Mineviciute, D. Hertel, R. Wellander, A. Yartsev and V. Sundstrom, J. Chem. Phys., 2007, 127, 144907. 
37 J.-L. Brédas and R. Silbey, Science, 2009, 323, 348-349.

38 E. Mutlugun, P. L. Hernandez-Martinez, C. Eroglu, Y. Coskun, T. Erdem, V. K. Sharma, E. Unal, S. K. Panda, S. G. Hickey, N. Gaponik, A. Eychmüller and H. V. Demir, Nano Lett., 2012, 12, 3986-3993.

39 S. Nizamoglu, P. Ludwig Hernández-Martínez, E. Mutlugun, D. Ugur Karatay and H. Volkan Demir, Appl. Phys. Lett., 2012, 100, 241109.

40 P. L. Hernández-Martínez, A. O. Govorov and H. V. Demir, J. Phys. Chem. C, 2013, 117, 10203-10212.
41 S. Nizamoglu, B. Guzelturk, D.-W. Jeon, I.-H. Lee and H. V. Demir, Appl. Phys. Lett., 2011, 98, 163108.

42 C. Kagan, C. Murray, M. Nirmal and M. Bawendi, Phys. Rev. Lett., 1996, 76, 1517-1520.

43 M. Artemyev, A. Bibik, L. Gurinovich, S. Gaponenko and U. Woggon, Phys. Rev. B: Condens. Matter Mater. Phys, 1999, 60, 1504-1506.

44 L. Romaner, A. Pogantsch, P. Scandiucci de Freitas, U. Scherf, M. Gaal, E. Zojer and E. J. W. List, Adv. Funct. Mater., 2003, 13, 597-601. 